\title{
Vortices and extreme black holes: The question of flux expulsion
}

\author{
Filipe Bonjour, ${ }^{*}$ Roberto Emparan, ${ }^{\dagger}$ and Ruth Gregory ${ }^{\dagger}$ \\ Centre for Particle Theory, Durham University, South Road, Durham, DH1 3LE, United Kingdom
}

(Received 29 October 1998; published 24 March 1999)

\begin{abstract}
It has been claimed that extreme black holes exhibit a phenomenon of flux expulsion for Abelian Higgs vortices, irrespective of the relative width of the vortex to the black hole. Recent work by two of the authors showed a subtlety in the treatment of the event horizon, which cast doubt on this claim. We analyze in detail the vortex-extreme black hole system, showing that, while flux expulsion can occur, it does not do so in all cases. We give analytic proofs for both expulsion and penetration of flux, in each case deriving a bound for that behavior. We also present extensive numerical work backing up, and refining, these claims, and showing in detail how a vortex can end on a black hole in all situations. We also calculate the back reaction of the vortex on the geometry, and comment on the more general vortex-black hole system. [S0556-2821(99)04608-1]
\end{abstract}

PACS number(s): 04.70.Dy, 11.27.+d

\section{INTRODUCTION}

The story of black hole hair is an ongoing and interesting one. It was thought for some time that black holes were relatively bland objects, classified by very few parameters: charge, mass, and angular momentum. This picture has changed significantly in the past decade with the discovery of various types of solutions which carry other, more exotic, charges — such as the colored black holes [1]—and solutions with dressed horizons [2]. What is clear is that when a nontrivial topology is allowed for the matter fields, so-called "no-hair' theorems can often be evaded. In this paper, we are interested in the question of Abelian Higgs hair, which may occur because a U(1) vortex can pierce, or even end, on a black hole [3]. This particular phenomenon is interesting both from the point of view of hair for the black hole as well as providing a decay channel for the disintegration of otherwise stable topological defects [4-8].

Briefly, the results of [3] showed that is was possible for a vortex solution of the $\mathrm{U}(1)$ Abelian Higgs model-a Nielsen-Olesen vortex [9] — to thread a Schwarzschild black hole, and that the matter fields reacted very little to the presence of the event horizon. Inclusion of back reaction of the vortex on the geometry revealed that it was an appropriate smooth version of the Aryal-Ford-Vilenkin geometry [10] discovered some years previously. Further work [7] showed that the conical singularities in other more complicated geometries could be smoothed over by the vortex, which allows the exact vacuum instanton for the splitting of a conical defect [11] to be used to construct a smooth instanton for splitting of physical topological defects [4-6]. A technical feature of these smooth gravitational instantons was that they contained two U(1) fields, the broken $\mathrm{U}(1)$ of the Abelian Higgs vortex, and an unbroken U(1), electromagnetism, needed to give a regular Euclidean section for the instanton (although not needed for regularity of the Lorentzian section). Naturally this raised the question as to whether the

\footnotetext{
*Email address: Filipe.Bonjour@durham.ac.uk

${ }^{\dagger}$ Email address: Roberto.Emparan@durham.ac.uk

\#Email address: R.A.W.Gregory@durham.ac.uk
}

results of [3] were still valid in the presence of this extra field. In the papers of Chamblin et al. [12] it was argued that while the results of [3] were qualitatively the same for nonextremal black holes, in the extremal limit a completely new phenomenon occurred, and the flux of the vortex was expelled from the black hole, rather like flux is expelled from a superconductor.

The evidence presented in [12] was of the form of analytic arguments for high winding vortices, and numerical work representing a black hole-vortex system for a variety of relative sizes of black hole to the string in which the flux lines of the vortex appeared to consistently wrap the black hole. In a previous Comment [13], two of us pointed out that there were some difficulties with the numerical evidence as stated, and that while expulsion was possible for thick, or high winding, vortices, it did not appear to occur for thin vortices. This would apparently solve a puzzle noted by Chamblin et al., namely that one could, in principle, take a vortex terminating on a near-extreme black hole (see [14] for a detailed discussion of selection rules for terminating vortices) and then charge the black hole up to extremality. This would appear to be in contradiction with the principle of flux expulsion. However, if flux expulsion is not mandatory, then such a puzzle never arises. There is, however, another problem if flux is always expelled. It appears that very thin vortices have a higher energy wrapping the black hole than if there were no black hole present at all. This would mean that the system is unstable and the string expels the black hole, which then raises a physical paradox. A string outside a black hole reacts to the black hole's gravitational field, therefore we might expect it to be attracted. On the other hand, the vortex is not charged under the massless $U(1)$, therefore it has no reason to feel any repulsion, except for the putative flux expulsion force. It is therefore not easy to see what the equilibrium solution of a vortex-black-hole system would be. Of course this is a rather naive argument, as it ignores any effects due to the conical deficit geometry of a gravitating cosmic string, which does produce a repulsive force on charges [15], as well as an attractive force on masses [16], a point which we return to in the conclusion after we have explored the issue of gravitational back reaction of the vortex. 
In this paper, we submit the Abelian Higgs vortexextreme black hole system to an exhaustive analysis, with the intent of pinning down precisely when, or indeed whether, flux expulsion can or cannot occur. We give analytic arguments for flux expulsion in certain regions of parameter space, and flux penetration in others, and back up the analysis with a wealth of numerical data. We discuss the problems of numerical integration of this system (and how our work differs from that presented in [12]) and how we have avoided these and ensured accuracy of the integrations. We also consider in detail the vortex terminating on the black hole. We then include a discussion of gravitational back reaction before concluding.

\section{THE ABELIAN HIGGS VORTEX}

We start by reviewing the $\mathrm{U}(1)$ vortex in order to establish notation and conventions. The action for an Abelian Higgs system is

$$
\begin{aligned}
S_{1}= & \int d^{4} x \sqrt{-g}\left[D_{\mu} \Phi^{\dagger} D^{\mu} \Phi\right. \\
& \left.-\frac{1}{4} \widetilde{G}_{\mu \nu} \widetilde{G}^{\mu \nu}-\frac{1}{4} \lambda\left(\Phi^{\dagger} \Phi-\eta^{2}\right)^{2}\right],
\end{aligned}
$$

where $\Phi$ is a complex scalar field, $D_{\mu}=\nabla_{\mu}+i e B_{\mu}$ is the usual gauge covariant derivative, and $\widetilde{G}_{\mu \nu}$ the field strength associated with $B_{\mu}$. We use units in which $\hbar=c=1$ and a mostly minus signature. It is conventional to express the field content in a slightly different manner in which the physical degrees of freedom are made more manifest by defining real fields $X, \chi$ and $P_{\mu}$ by

$$
\begin{aligned}
\Phi\left(x^{\alpha}\right) & =\eta X\left(x^{\alpha}\right) e^{i \chi\left(x^{\alpha}\right)} \\
B_{\mu}\left(x^{\alpha}\right) & =\frac{1}{e}\left[P_{\mu}\left(x^{\alpha}\right)-\nabla_{\mu} \chi\left(x^{\alpha}\right)\right] .
\end{aligned}
$$

These fields represent the physical degrees of freedom of the broken symmetric phase; $X$ is the scalar Higgs field, $P_{\mu}$ the massive vector boson, and $\chi$, being a gauge degree of freedom, is not a local observable, but can have a globally nontrivial phase factor which indicates the presence of a vortex. The existence of vortex solutions in the Abelian Higgs model was argued by Nielsen and Olesen [9], and in the presence of a vortex $\oint d \chi=2 \pi N$, where $N$ is the winding number of the vortex.

In terms of these new variables, the equations of motion are

$$
\begin{array}{r}
\nabla_{\mu} \nabla^{\mu} X-P_{\mu} P^{\mu} X+\frac{\lambda \eta^{2}}{2} X\left(X^{2}-1\right)=0 \\
\nabla_{\mu} G^{\mu \nu}+\frac{X^{2} P^{\nu}}{\beta}=0,
\end{array}
$$

where $\beta=\lambda / 2 e^{2}$ is the Bogomol'nyi parameter [17], and $G_{\mu \nu}$ is the field strength of $P_{\mu}$.

The simplest possible vortex solution is that in flat space:

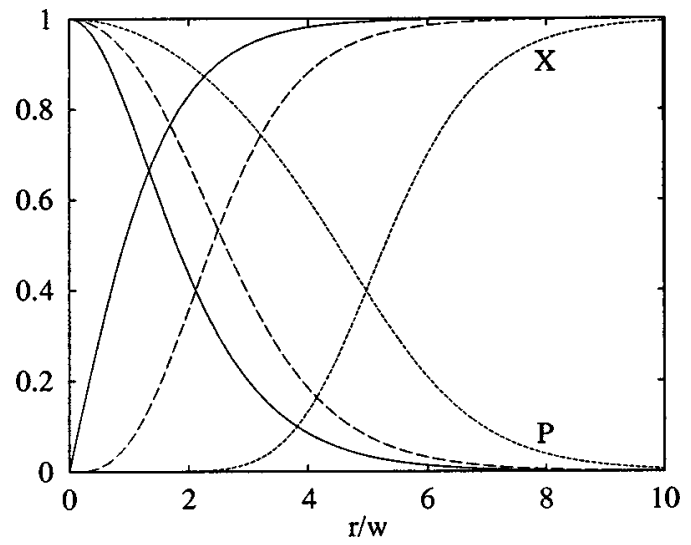

FIG. 1. The Nielsen-Olesen vortex for $\beta=1$ and for $N=1$ (solid lines), $N=3$ (long-dashed lines) and $N=10$.

$$
X=X(R), \quad P_{\mu}=N P(R) \partial_{\mu} \phi,
$$

where $R=r \sqrt{\lambda} \eta,\{r, \phi\}$ are polar coordinates, and $X$ and $P$ satisfy the coupled second order ordinary differential equations (ODE's)

$$
\begin{array}{r}
-X^{\prime \prime}-\frac{X^{\prime}}{R}+\frac{X N^{2} P^{2}}{R^{2}}+\frac{1}{2} X\left(X^{2}-1\right)=0 \\
-P^{\prime \prime}+\frac{P^{\prime}}{R}+\frac{X^{2} P}{\beta}=0 .
\end{array}
$$

For $N=1$, this is the Nielsen-Olesen solution, and gives an isolated vortex for all $\beta$. The vortex core consists of two components-a scalar core where the Higgs field differs from vacuum, roughly of width $1 / \sqrt{\lambda} \eta$, and a gauge core of thickness $\mathrm{O}\left(\beta^{1 / 2} / \sqrt{\lambda} \eta\right)$. For higher $N$, the solutions were given in [18], the principal differences to $N=1$ being that the $X$-field is flattened $\left(X \sim R^{N}\right)$ near the core, and the string is correspondingly fattened. An additional difference is that for $\beta>1$, higher winding strings are unstable to separation into $N$ unit winding vortices [17]. Figure 1 presents some solutions for $\beta=1$.

In this paper however, we are interested in nontrivial solutions in curved space, specifically in the presence of a charged black hole. This means that our set-up now has three length scales, the two string core widths as already mentioned, and the new scale-the black hole's outer horizon radius:

$$
\begin{aligned}
w_{\mathrm{Higgs}} \sim m_{\mathrm{Higgs}}^{-1} & =\frac{1}{\sqrt{\lambda} \eta} \\
w_{\text {gauge }} \sim m_{\text {gauge }}^{-1} & =\frac{1}{\sqrt{2} e \eta} \\
r_{H} & =G m+\sqrt{G^{2} m^{2}-G q^{2}}
\end{aligned}
$$

where $m$ is the Arnowitt-Deser-Misner (ADM) mass of the black hole, and $q$ its charge $\left(A_{0}=q / r\right)$. We now set $\lambda \eta^{2}$ $=1$, which considerably streamlines our analysis and equa- 
tions of motion. The gauge width of the core has already been replaced by $\sqrt{\beta}$, and we now replace the mass and charge of the Reissner-Nordstr $\varnothing m$ black hole by $M$ $=G m / w_{H}$ and $Q^{2}=G q^{2} / w_{H}^{2}$. This simply means that we have chosen to set the Higgs mass, rather than the Planck mass, to unity. There are now no dimensionful quantities, and Newton's constant, $G$, is a (small) number which we will represent by $\epsilon$, where $\epsilon=8 \pi G \eta^{2}$. For a grand unified theory (GUT) string $\epsilon=\mathrm{O}\left(10^{-6}\right)$, and represents the gravitational strength of the string, which will be used in Sec. VI, when we consider the gravitational back reaction of the vortex.

For now, however, we ignore the gravitational back reaction of the vortex and, as in [12], treat the vortex in the background Reissner-Nordstrom geometry:

$$
\begin{aligned}
d s^{2}= & \left(1-\frac{2 M}{r}+\frac{Q^{2}}{r^{2}}\right) d t^{2}-\left(1-\frac{2 M}{r}+\frac{Q^{2}}{r^{2}}\right)^{-1} d r^{2} \\
& -r^{2}\left[d \theta^{2}+\sin ^{2} \theta d \phi^{2}\right] .
\end{aligned}
$$

For the moment, it is irrelevant whether the geometry is the result of an electric or magnetic potential since we only require the equations for the vortex fields in the ReissnerNordstrøm geometry. Substituting Eq. (7) in Eqs. (3a), (3b), and assuming the form (4) for the $P_{\mu}$-field gives

$$
\begin{array}{r}
-\frac{1}{r^{2}} \partial_{r}\left(\left(r^{2}-2 M r+Q^{2}\right) \partial_{r} X\right)-\frac{1}{r^{2} \sin \theta} \partial_{\theta}\left(\sin \theta \partial_{\theta} X\right) \\
+\frac{X N^{2} P^{2}}{r^{2} \sin ^{2} \theta}+\frac{1}{2} X\left(X^{2}-1\right)=0 \\
\partial_{r}\left[\left(1-\frac{2 M}{r}+\frac{Q^{2}}{r^{2}}\right) \partial_{r} P\right]+\frac{\sin \theta}{r^{2}} \partial_{\theta}\left[\frac{\partial_{\theta} P}{\sin \theta}\right]-\frac{X^{2} P}{\beta}=0 .
\end{array}
$$

In general, these equations are intractable analytically, however, as in [3] we can extract some information in a particular limit—the "thin string limit" where we assume $M \gg 1$; the value of $Q$ is irrelevant. First note that if we write $R$ $=r \sin \theta$, and make the assumption that $X=X(R), P$ $=P(R)$, then Eq. (8) becomes

$$
\begin{aligned}
-X^{\prime \prime} & -\frac{X^{\prime}}{R}+\frac{X N^{2} P^{2}}{R^{2}}+\frac{1}{2} X\left(X^{2}-1\right) \\
& =\frac{R^{2}}{r^{2}}\left(\frac{2 M}{r}-\frac{Q^{2}}{r^{2}}\right)\left[X^{\prime \prime}+\frac{X^{\prime}}{R}\right] \\
-P^{\prime \prime}+\frac{P^{\prime}}{R}+\frac{X^{2} P}{\beta} & =\frac{R^{2}}{r^{2}}\left(\frac{Q^{2}}{r^{2}}\left(P^{\prime \prime}-2 \frac{P^{\prime}}{R}\right)-\frac{2 M}{r}\left(P^{\prime \prime}-\frac{P^{\prime}}{R}\right)\right] .
\end{aligned}
$$

However, note that the right-hand side (RHS) of each of these equations has the form $R^{2} / r^{2}$ times terms of order unity. Near the core $R$ is order unity, hence the RHS is $\mathrm{O}\left(M^{-2}\right)$, thus we have the flat space equations (5a), (5b) for which the solutions are well known, and have the property that away from the core the vortex fields tend to their vacuum values exponentially rapidly. Therefore, by the time the premultiplying term in the RHS of Eqs. (9a),(9b) becomes significant, the fields are essentially in vacuum, so any corrections will be negligible. We can therefore regard the flat space solutions (as functions of $R=r \sin \theta$ ) as a good approximation to the true solution in the thin string limit. Note that this form of the solution pierces the horizon and does not depend on the value of $Q$, therefore, using this argument, one would expect that thin strings always penetrate the event horizon of a black hole, whether or not it is extremal.

The argument developed so far starts from the thin string limit, but there is another limit in which considerable information can be extracted analytically, and which leads to the expectation that vortices are expelled from extremal horizons. This regime, which can be regarded as a "thick string limit" complementary to the one above, is attained for large winding number $N$. As shown in [18], when $N$ is large the size of the vortex grows like $\sqrt{N}$, and the unbroken phase inside the core is approached increasingly faster, $X \sim R^{N}$. Consider then a black hole that sits well inside the vortex core. There, the field is expected to be very close to the symmetric phase, so it seems reasonable to neglect the last term in Eq. (8b). Then the equation can be solved by

$$
P \approx 1-p\left(r^{2}-Q^{2}\right) \sin ^{2} \theta,
$$

with $p \approx 1 /(2 N \sqrt{\beta})$ [12]. From here we see that, in the extremal limit in which the horizon is at $r=Q$, the magnetic flux across the horizon, given by $G_{\theta \phi}=\partial_{\theta} P$, vanishes. Moreover, it is possible to solve for the Higgs field $X$ by setting $X=[b(r) \sin \theta]^{N}$, and keeping only leading terms in $1 / N$. One finds, near the horizon,

$$
d(\log b) \propto \frac{d r}{\sqrt{r^{2}-2 M r+Q^{2}}}=\frac{d r}{\sqrt{\left(r-r_{+}\right)\left(r-r_{-}\right)}},
$$

so that, if the black hole is extremal $\left(r_{+}=r_{-}\right)$then $b \sim r$ $-r_{+}$. Hence, $X$ vanishes as well on the extremal horizon. Furthermore, a study of the energy of the configuration shows that it is favorable for the black hole to remain inside the vortex core [12].

Indeed, the behavior of magnetic fields in the vicinity of extremal horizons has been studied in more generality in [19] and the expulsion of the flux-a phenomenon remarkably analogous to the Meissner effect in superconductors-has been argued to be generic. Extremal horizons tend to repel magnetic fields, at least if the latter are in, or approach, a phase of unbroken symmetry, like in the core of the vortex. The argument above for large $N$ vortices, however, is not fully conclusive, since it remains the possibility that corrections of higher order in $M$ or in $1 / N$ spoil the exact expulsion phenomenon.

\section{ANALYTIC ARGUMENTS AND BOUNDS}

In the previous section we summarized the arguments of [12] in favor of flux expulsion, and extended the thin string 
arguments of [3] which seemed to indicate flux penetration. Both of these arguments appear compelling, and we must examine the system closely to see what definite information can be extracted. First, note that all methods are in agreement that there is flux penetration for nonextremal black holes, therefore for the rest of this section, we will only be considering extremal black holes, for which the metric is

$d s^{2}=\left(1-\frac{M}{r}\right)^{2} d t^{2}-\left(1-\frac{M}{r}\right)^{-2} d r^{2}-r^{2} d \theta^{2}-r^{2} \sin ^{2} \theta d \phi^{2}$.

This gives the vortex field equations

$$
\begin{gathered}
-\frac{1}{r^{2}} \partial_{r}\left[(r-M)^{2} \partial_{r} X\right]-\frac{1}{r^{2} \sin \theta} \partial_{\theta}\left(\sin \theta \partial_{\theta} X\right) \\
+\frac{X N^{2} P^{2}}{r^{2} \sin ^{2} \theta}+\frac{1}{2} X\left(X^{2}-1\right)=0 \\
\partial_{r}\left[\left(1-\frac{M}{r}\right)^{2} \partial_{r} P\right]+\frac{\sin \theta}{r^{2}} \partial_{\theta}\left[\frac{\partial_{\theta} P}{\sin \theta}\right]-\frac{X^{2} P}{\beta}=0
\end{gathered}
$$

which as before are not analytically soluble, however, we can extract quite a bit of information about the solutions due to the nature of the geometry near $r=M$.

The first observation is that if we set $r=M$, then the equations for the horizon actually decouple from the exterior geometry:

$$
\begin{array}{r}
-\frac{1}{\sin \theta} \partial_{\theta}\left(\sin \theta \partial_{\theta} X\right)+\frac{X N^{2} P^{2}}{\sin ^{2} \theta}+\frac{1}{2} M^{2} X\left(X^{2}-1\right)=0 \\
\sin \theta \partial_{\theta}\left[\frac{\partial_{\theta} P}{\sin \theta}\right]-\frac{M^{2} X^{2} P}{\beta}=0,
\end{array}
$$

a phenomenon which does not occur in the nonextremal case. This means that the vortex equations on the horizon are now ODE's and therefore easier to handle. Note that the flux expulsion solution $(X=0, P=1)$ always solves Eqs. (14a), (14b), therefore we cannot use any analysis of these equations to demonstrate flux expulsion, but we can potentially show the nonexistence of a penetration solution.

Therefore, assume that a piercing solution to the vortex equations does exist throughout the spacetime, this means that a piercing solution must exist on the horizon. This requires a nontrivial solution $[X(\theta), P(\theta)]$ which is symmetric around $\theta=\pi / 2$ at which point $X$ has a maximum and $P$ a minimum. Let $X_{m}$ and $P_{m}$ be the extremal values of $X$ and $P$ attained. In addition, expanding Eqs. (14a),(14b) near the poles indicates that $P_{, \theta}=0$ at the poles. Therefore, there exists a $\theta_{0}(<\pi / 2)$ such that $P_{, \theta \theta}=0$ at $\theta_{0}$, and $P_{, \theta}\left(\theta_{0}\right)<0$.

The structure of the proof is as follows; we use the properties of the solution at $\pi / 2$ to derive an upper bound on $P$ and $P_{, \theta \theta}$ there. Then we use the behavior of $P_{, \theta}$ to derive a lower bound on $P_{, \theta \theta}$. At the very least, these must be consistent for a piercing solution to exist, therefore, if the inequalities are incompatible we conclude that the core solution is the only solution on the horizon and therefore flux expulsion must occur.

To provide the upper bound, consider the $X$ equation at $\theta=\pi / 2$. Since $X_{, \theta \theta}<0$, Eq. (14a) implies $P_{m}^{2}<\frac{1}{2}\left(M^{2} /\right.$ $\left.N^{2}\right)\left(1-X_{m}^{2}\right)<\frac{1}{2}\left(M^{2} / N^{2}\right)$ and hence

$$
P_{, \theta \theta}(\pi / 2)=\frac{M^{2}}{\beta} X_{m}^{2} P_{m}<\frac{M^{3}}{\sqrt{2} \beta N} X_{m}^{2}\left(1-X_{m}^{2}\right)^{1 / 2}<\frac{\sqrt{2}}{3 \sqrt{3}} \frac{M^{3}}{\beta N},
$$

where the final inequality is obtained by maximizing over $X_{m}$.

For the lower bound, on the other hand note that at $\theta_{0}\left|P_{, \theta}\right|$ takes its largest value, $\left|P_{, \theta_{0}}\right|=\left(M^{2} / \beta\right) X^{2} P \tan \theta_{0}$ $<\left(M^{2} / \beta\right) \tan \theta_{0}$, and hence

$$
\frac{M}{\sqrt{2} N}>P_{m}>1-\frac{\pi}{2}\left|P_{, \theta_{0}}\right| \Rightarrow\left|P_{, \theta_{0}}\right|>\frac{2}{\pi}\left(1-\frac{M}{\sqrt{2} N}\right) .
$$

Assuming $\quad M<\sqrt{2} N$, this gives $\pi / 2-\theta_{0} \leqslant \cot \theta_{0}$ $<\pi M^{2} / 2 \beta(1-M / \sqrt{2} N)$. But then for $M^{2} X_{m}^{2}<2 \beta$ one can show that $P_{, \theta \theta}$ has a maximum at $\pi / 2$, hence

$$
P_{, \theta \theta}(\pi / 2) \geqslant \frac{P_{, \theta}(\pi / 2)-P_{, \theta}\left(\theta_{0}\right)}{\pi / 2-\theta_{0}}>\left(\frac{2}{\pi}\right)^{2} \frac{\beta}{M^{2}}\left(1-\frac{M}{\sqrt{2} N}\right)^{2} .
$$

Therefore, by comparing Eqs. (15) and (17), we see that an absolute minimum requirement for a piercing solution is the consistency of these two bounds, i.e.

$$
\sqrt{2} \pi^{2} M^{5}>12 \sqrt{3} \beta^{2} N\left(1-\frac{M}{\sqrt{2} N}\right)^{2}
$$

Turning this around therefore, and writing $\mathcal{M}=M / \sqrt{2} N$, we may conclude that the vortex flux lines must be expelled from an extreme Reissner-Nordstrøm black hole if

$$
\frac{\mathcal{M}^{5}}{(1-\mathcal{M})^{2}}<\frac{3 \sqrt{3}}{2 \pi^{2}} \frac{\beta^{2}}{N^{4}} \simeq \frac{\beta^{2}}{4 N^{4}}
$$

For $N=\beta=1$, this gives $M<0.7$; note that this is a rather weak bound, in fact we would expect flux expulsion to be mandatory for $M$ somewhat in excess of 0.7 , but this method at least provides an analytic proof giving a definite bound for $M$. Numerical work (Sec. IV) actually places this bound at about 1.9 (see Fig. 4).

It is interesting to note the variation of this bound with $N$ and $\beta$. For large $N, \mathcal{M}^{5}<O\left(N^{-4}\right)$, or $M<O\left(N^{1 / 5}\right)$. This means that the larger $N$, the larger the black hole can be and still have flux expulsion. This is in agreement with the observation that large $N$ vortices are thicker than their single winding number counterparts, therefore we would expect 
flux expulsion to occur more readily. Indeed, this is how [12] originally argued for flux expulsion.

For $\beta \rightarrow 0$ we see that $M \leqslant O\left(\beta^{2 / 5}\right)$, i.e., that flux expulsion only occurs for extremely small black holes. To understand this, recall that the fall-off of the $P$ field is $P$ $\simeq e^{-R / \sqrt{\beta}}$ therefore as $\beta \rightarrow 0$, the magnetic flux core of the string is getting smaller as $\sqrt{\beta}$. This is consistent with the above bound. It is therefore interesting to look at large $\beta$, since in this limit, the magnetic core becomes very diffuse, and we are left with the Higgs core, which is no longer exponentially cut-off, but follows a power law cut-off approaching that of the global string. For large $\beta$, the bound (18) gives $\mathcal{M}<1-O\left(\beta^{-2}\right)$, hence $M<\sqrt{2} N+O\left(\beta^{-2}\right)$. Therefore, for small charge Higgs scalars, or global strings, we expect flux expulsion to occur for $M$ of order the winding number of the string.

Having shown that flux expulsion must occur for sufficiently thick strings, what of the argument of the previous section, which appeared to indicate that a thin string would pierce the event horizon? Obviously, since the core is always a solution to the horizon system of equations, we cannot use an argument based on this system to argue flux penetration, but instead we must look at the full partial differential equation (PDE) system of equations in the exterior region of the horizon, Eqs. (13a),(13b).

Now, assume that there is a flux expulsion solution, then on $r=M, X=0$ and $P=1$. Therefore, near $r=M, M^{2} X^{2}$ $\ll 1$ and $\left[(r-M)^{2} X_{, r}\right]_{, r}>0$; hence

$$
\frac{1}{2} M^{2} X \sin ^{2} \theta+\sin \theta \partial_{\theta} \sin \theta \partial_{\theta} X<X N^{2} P^{2}<X N^{2}
$$

in this region. Now, we know that $X$ is symmetric around $\pi / 2$, peaking at some maximum $X_{m}$, and also that $\sin \theta \partial_{\theta} X$ vanishes at $0, \pi / 2$ and $\pi$; therefore let $\theta_{0}$ be the value at which $\partial_{\theta} \sin \theta \partial_{\theta} X=0$, which must satisfy $\frac{1}{2} M^{2} \sin ^{2} \theta_{0}<N^{2}$. If $M<\sqrt{2} N$, then this inequality is clearly satisfied, so we now take $M>\sqrt{2} N$, and let $\alpha>\theta_{0}$ be defined by $M^{2} \sin ^{2} \alpha=2 N^{2}$.

Integrating Eq. (19) on the range $(\theta, \pi / 2)$, for $\theta>\alpha$ gives

$$
X_{, \theta}(\theta)>X(\theta)\left[\frac{1}{2} M^{2} \cot \theta+N^{2} \csc \theta \ln \tan \theta / 2\right] .
$$

But since $X_{, \theta \theta}<0$ on $\left[\theta_{0}, \pi / 2\right]$, we can deduce $X_{, \theta}(\theta)$ $<\left[X(\theta)-X\left(\theta_{0}\right)\right] /\left(\theta-\theta_{0}\right)<X(\theta) / \theta-\alpha$, hence for consistency with Eq. (20)

$$
\frac{1}{N^{2}}>(\theta-\alpha)\left[\csc ^{2} \alpha \cot \theta+\csc \theta \ln \tan \theta / 2\right]
$$

must hold over the range $\theta \in(\alpha, \pi / 2)$ for the expulsion solution to hold. The actual bound on $M$ is then obtained by plotting these curves and determining for which $M$ this inequality is always satisfied. For $N=\beta=1$ we find that for $M^{2}>8.5$, this inequality is violated, hence the vortex must pierce the horizon in this case.

For larger $N$, the lower bound on $M$ for a piercing solution to be forced does increase, however, the ratio $M / N$ ac- tually decreases. The LHS of Eq. (21) is $N^{-2}$ and it is easy to see that this requires $\alpha=\pi / 2-O\left(N^{-1 / 2}\right)$. From this, we therefore obtain $M>\sqrt{2} N\left[1+O\left(N^{-1}\right)\right]$. Note that this argument does not depend on $\beta$. This means that for large $\beta$ we still get piercing solutions for the same range of $M$. Since $\beta \rightarrow \infty$ corresponds to the global string, this should not be too surprising. For $\beta \rightarrow 0$, although we expect some drop in the value of $M$, this method is unable to detect this.

When the vortex radius is much larger than the black hole radius one can find approximate explicit solutions for the fields near the horizon. Indeed, one can construct these solutions for arbitrary winding number $N$, therefore generalizing the solutions in [12].

The solution for the magnetic field is readily found by noticing that if $M$ (or better, $M / \sqrt{\beta}$ ) is very small, then inside the vortex and close to the horizon the gauge field is well approximated by the solution to the massless field equation $[12,19]$

$$
P \simeq 1-2 M p(r-M) \sin ^{2} \theta,
$$

where $p$ is an integration constant equal to twice the magnetic field strength at the center of the core. Next, by looking at the Higgs field equation, we can see that close to the horizon the potential term $\frac{1}{2} X\left(X^{2}-1\right)$ is suppressed by a factor $M^{2}$ which we are taking to be small. Therefore it can be neglected. After setting $P \approx 1$, the resulting equation can be solved with $X$ a function exclusively of $(r-M) \sin \theta$,

$$
X \simeq k[(r-M) \sin \theta]^{N},
$$

where $k$ is another integration constant. We see that on the horizon both the gauge and the Higgs field are expelled. Comparison between these approximate solutions and the results of the numerical calculations in the next section shows good agreement.

\section{NUMERICAL RESULTS}

To solve Eqs. (8a), (8b) numerically, we have used the technique developed in [3], which consists of relaxing initial configurations of the fields $X$ and $P$ on the (rectangularly) discretized plane, $(r, \theta) \rightarrow\left(r_{i}=r_{+}+i d r, \theta_{j}=j d \theta\right)$. We therefore replace the fields by their values on this grid, $X(r, \theta)$ $\rightarrow X_{i, j} \equiv X\left(r_{i}, \theta_{j}\right)$ (and similarly for $P$ ), and the differential operators by suitably discretized versions. Adopting the notation of [3] and [12] (that is, $X_{00}=X_{i, j}, X_{ \pm 0}=X_{i \pm 1, j}$ and $\left.X_{0 \pm}=X_{i, j \pm 1}\right)$, we find that the discretized version of Eqs. (8a),(8b) is $X_{00} \rightarrow X_{00}^{\text {new }}, P_{00} \rightarrow P_{00}^{\text {new }}$, where 


$$
\begin{aligned}
X_{00}^{\text {new }}= & \frac{\frac{2}{r}\left(1-\frac{M}{r}\right) \frac{X_{+0}-X_{-0}}{2 \Delta r}+\frac{\cot \theta}{r^{2}} \frac{X_{0+}-X_{0-}}{2 \Delta \theta}+\left(1-\frac{2 M}{r}+\frac{Q^{2}}{r^{2}}\right) \frac{X_{0+}+X_{0-}}{\Delta r^{2}}+\frac{X_{0+}+X_{0-}}{r^{2} \Delta \theta^{2}}}{\left(1-\frac{2 M}{r}+\frac{Q^{2}}{r^{2}}\right) \frac{2}{\Delta r^{2}}+\frac{2}{r^{2} \Delta \theta^{2}}+\frac{1}{2}\left(X_{00}^{2}-1\right)+\left(\frac{N P_{00}}{r \sin \theta}\right)^{2}} \\
P_{00}^{\text {new }}= & \frac{\frac{2}{r^{2}}\left(M-\frac{Q^{2}}{r}\right) \frac{P_{+0}-P_{-0}}{2 \Delta r}-\cot \theta \frac{P_{0+}-P_{0-}}{2 r^{2} \Delta \theta^{2}}+\left(1-\frac{2 M}{r}+\frac{Q^{2}}{r^{2}}\right) \frac{P_{0+}+P_{0-}}{\Delta r^{2}}+\frac{P_{0+}+P_{0-}}{r^{2} \Delta \theta^{2}}}{\left(1-\frac{2 M}{r}+\frac{Q^{2}}{r^{2}}\right) \frac{2}{\Delta r^{2}}+\frac{2}{r^{2} \Delta \theta^{2}}+\frac{X_{00}^{2}}{\beta}} .
\end{aligned}
$$

There is, however, a subtlety in this process: relaxation methods usually require that the values of the fields be fixed at all the boundaries of the domain of integration, and although we know the asymptotic values of $X$ and $P$ at $r \rightarrow \infty$ (the vacuum) and at $\theta \rightarrow 0, \pi$ (the string core values), the configuration at the horizon $r=r_{+}$is in fact the main result we expect from this numerical calculation. The solution to this problem conceived in [3] was to update the values of the fields at the horizon immediately after updating the interior of the grid. Note that this still requires an initial guess for the fields on the horizon - a crucial point we will return to later. Replacing $r=r_{+}$in Eqs. (8a),(8b) we obtain equations on the horizon:

$$
\begin{aligned}
\left.\frac{r_{+}-r_{-}}{r_{+}^{2}} \frac{\partial X}{\partial r}\right|_{r=r_{+}}= & -\frac{1}{r_{+}^{2} \sin \theta} \partial_{\theta}\left(\sin \theta \partial_{\theta} X\right) \\
& +\frac{1}{2} X\left(X^{2}-1\right)+\frac{N^{2} X P^{2}}{r_{+}^{2} \sin ^{2} \theta} \\
\left.\frac{r_{+}-r_{-}}{r_{+}^{2}} \frac{\partial P}{\partial r}\right|_{r=r_{+}}= & -\frac{\sin \theta}{r_{+}^{2}} \partial_{\theta}\left(\frac{\partial_{\theta} P}{\sin \theta}\right)+\frac{X^{2} P}{\beta}
\end{aligned}
$$

clearly, these equations reduce to Eqs. (14a),(14b) in the extremal case. We discretize this in the same way that we discretized the equations on the interior of the grid (except that we must now take discretized differential operators that do not depend on $X_{-0}$ or $P_{-0}$ ). The resulting equations are

$$
\begin{aligned}
X_{00} \rightarrow X_{00}^{\text {new }} & \sqrt{M^{2}-Q^{2}} \frac{X_{+0}}{\Delta r}+\frac{X_{0+}+X_{0-}}{2 \Delta \theta^{2}}+\cot \theta \frac{X_{0+}-X_{0-}}{4 \Delta \theta} \\
= & \frac{\sqrt{M^{2}-Q^{2}}}{\Delta r}+\frac{1}{\Delta \theta^{2}}+\frac{r_{+}}{4}\left(X_{00}^{2}-1\right)+\frac{1}{2}\left(\frac{N P_{00}}{\sin \theta}\right)^{2}
\end{aligned}
$$

$$
\begin{aligned}
P_{00} \rightarrow P_{00}^{\text {new }} \\
=\frac{\sqrt{M^{2}-Q^{2}} \frac{P_{+0}}{\Delta r}+\frac{P_{0+}+P_{0-}}{2 \Delta \theta^{2}}-\cot \theta \frac{P_{0+}-P_{0-}}{4 \Delta \theta}}{\frac{\sqrt{M^{2}-Q^{2}}}{\Delta r}+\frac{1}{\Delta \theta^{2}}+\frac{r+}{2 \beta} X_{00}^{2}} .
\end{aligned}
$$

The process of updating the interior of the grid and then the horizon at each iteration was carried on until the modulus of the largest relative correction on the grid became smaller than some $\varepsilon$ :

$$
\max _{i, j}\left|\frac{X_{i, j}^{\text {new }}-X_{i, j}^{\text {old }}}{X_{i, j}^{\text {old }}}\right|, \max _{i, j}\left|\frac{P_{i, j}^{\text {new }}-P_{i, j}^{\text {old }}}{P_{i, j}^{\text {old }}}\right|<\varepsilon .
$$

( $i$ and $j$ run over the entire grid, including the horizon.)

The results obtained by our implementation of this method were compared with the plots of [3], and we found a satisfactory agreement; for instance, Fig. 2 shows the contours of $X$ and $P$ for $M=10, \beta=1 / 2$ and $N=100$; it can be directly compared with Fig. 3 from [3]. $\left(r_{m}\right.$ is the maximum value for $r$, approximating $r \rightarrow \infty$.)
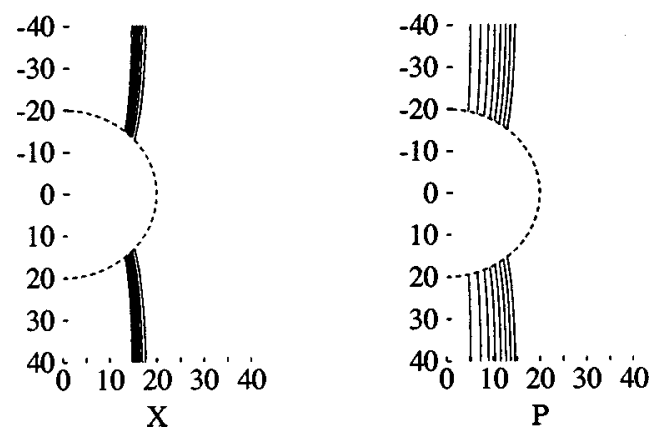

FIG. 2. Contours of $X$ and $P$ for $M=10, Q=0, \beta=1 / 2, N$ $=100, \varepsilon=10^{-4}$ and $N_{r}=N_{\theta}=r_{m}=100$. (The dashed semi-ellipse represents the horizon.) 

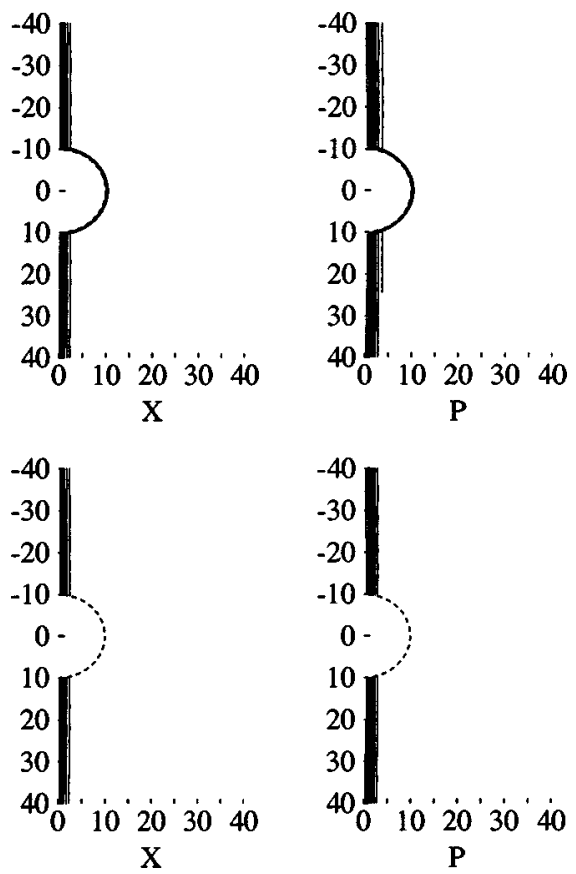

FIG. 3. Contours of $X$ and $P$ for a core guess (top) and a vacuum (or sinusoidal) initial guess. The parameters are $M=Q=10, \beta=N$ $=1, \varepsilon=10^{-4}, N_{r}=N_{\theta}=100$.

As in [3], we have found that the thin string approximation is excellent for thin vortices, and is a reasonable approximation even for thicker vortices, which tend to pinch slightly near the horizon.

We then turned our attention towards charged black holes, comparing now our results with those of Chamblin et al. [12]. We found, as they did, that in nonextremal cases the picture remains qualitatively the same as for uncharged black holes. For extremal black holes, however, our results differ from their original claims. As reported in [13], we find that the claimed expulsion of the matter fields for thin strings in this limit is the result of a loophole in the numerical method (when applied to extremal black holes), which does not take into consideration the decoupling of the horizon from the main grid. [This was shown in Eqs. (14a),(14b) and can also be seen from Eqs. (26a),(26b): if $Q=M$, these equations do not contain the terms $X_{+0}$ or $P_{+0}$.] In this case, the core

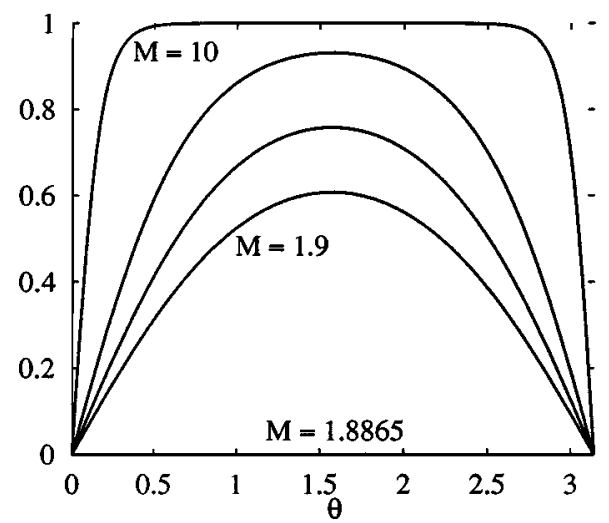

configuration is always an exact solution on the horizon; bearing in mind that the relaxation method updates the fields from a user-supplied initial guess, we see that if the initial guess made on the horizon is core, then the horizon will never be updated. In fact, [12] always started from this guess on the boundary, and therefore always obtained wrapping solutions in the extremal case.

Obviously, this choice of initial conditions is important, moreover, since we are dealing with a nonlinear system of PDE's, there is no reason for different initial configurations to relax to the same solution. For this reason, we have considered the following three initial data sets on the horizon:

Core: $X=0, P=1$,

Vacuum: $X=1, P=0$,

Sinusoidal: $X(\theta)=\sin \theta, P=1$.

(The sinusoidal guess was chosen because it interpolates smoothly, and in a simple and convenient way, the strings attached to the North and South poles of the black hole.)

Figure 3 displays the solutions obtained from the three initial configurations. By computing and comparing the energy densities and total energies of the fields on the grid, we were able to determine that inside the grid the three solutions were identical; on the horizon, however, the solution relaxed from the core guess was a string core, whereas the solutions obtained from the vacuum and the sinusoidal guesses were both the vacuum. The comparison also showed that the wrapping solution had a higher total energy than the piercing one; the difference is of course due to the jump of the fields from the horizon to the interior of the grid. (Making the stepsize $d r$ smaller failed to smooth out this sharp jump.)

To summarize, we have three (physical and numerical) reasons to prefer the piercing solution to the wrapping one for thin strings in the extremal limit: it is smooth, numerically more robust, and energetically favorable.

To determine how the transition from a piercing to a wrapping solution occurs as we thicken the string, we take advantage of the fact that, on the horizon, we now have ODE's. This allows for much quicker and more accurate numerical methods; for the following calculations we have used the relaxation routine SOLVDE of Ref. [20], Chap. 17, starting from a vacuum guess.

Figure 4 shows the solutions on the horizon for $\beta=N$ $=1$. For massive black holes (or, equivalently, thin strings),

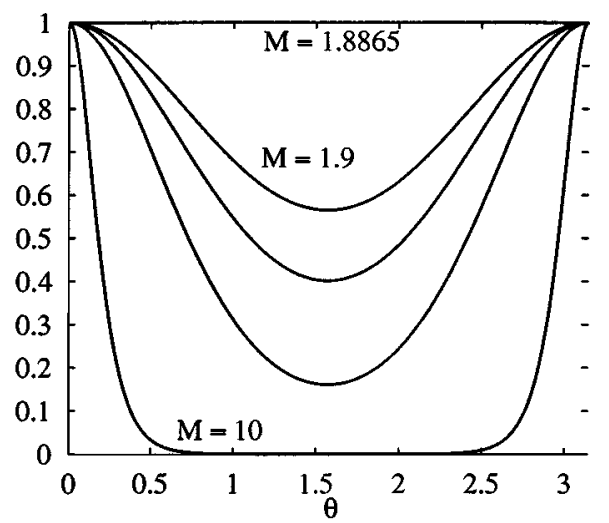

FIG. 4. Functions $X(\theta)$ (left) and $P(\theta)$ on the horizon for $\beta=N=1$, a vacuum guess and $M=10,2.5,2,1.9$ and 1.8865 . 

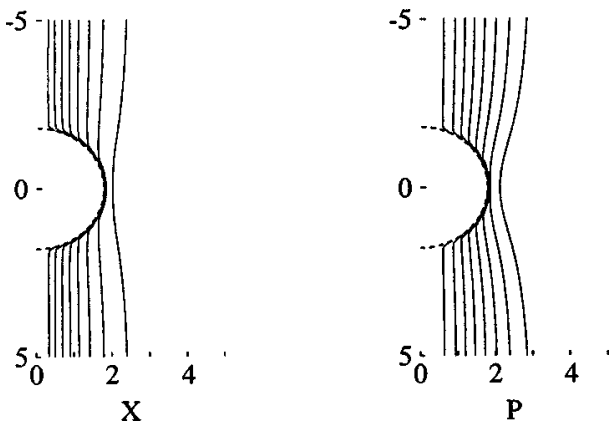

FIG. 5. Contours of $X$ and $P$ for $M=Q=1.8, N=\beta=1$, $\varepsilon=10^{-3}$ and $N_{r}=N_{\theta}=100$.

the fields adopt a vacuum profile on most of the horizon (symmetrically about the equator $\theta=\pi / 2$ ) and interpolate smoothly to their fixed core values on the poles. The shapes of $X$ and $P$ remain the same for all values of $M: X$ has non-vanishing $\theta$-derivatives at the poles, and a maximum at the equator; $P$ has zero derivatives at the poles, and a minimum at the equator. As we thicken the string, the maximum of $X$ and the minimum of $P$ move away from the vacuum values. For $N=\beta=1$ this transition is gentle at first, but accelerates suddenly, as if the string had crossed a critical width at which it is not able to pierce the horizon any more (see Fig. 5 for an example of expelled solution). This can be observed on Fig. 6, which shows the evolution of $X(\pi / 2)$ and $P(\pi / 2)$ with the inverse energy of the black hole for a variety of values of $\beta$. Increasing $\beta$, as we have remarked above, means thickening the $P$ tube, and we would therefore expect a heavier black hole to be required to expel this flux. This is indeed what we observe and, naturally, the curve for $P$ is more affected by this than that for $X$.

The same shift of the curves towards higher values of $M$ happens when one increases $N$, as anticipated from the fact that this also thickens the string. Figure 7 shows the evolution of the critical mass $M_{c}$ (defined to be that at which the horizon cannot support a penetrating solution any more) in function of $\beta$ (for $N=1$ ) and of $N($ for $\beta=1$ ).

\section{STRING ENDING ON A BLACK HOLE}

Next, we turn our attention to the case of a string ending on the black hole. This is an important configuration to con-

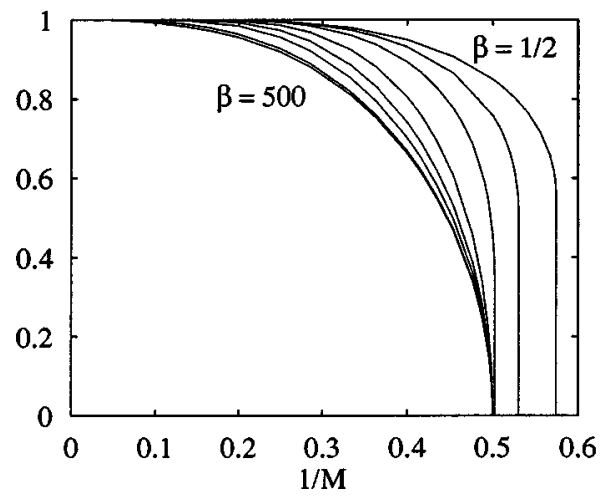

sider, since it is the main "phenomenological" input to the instantons mediating defect decay [4-8]. Originally, [12] supposed that such a configuration may not be able to exist, however, the thin string arguments indicate that at least for large $M$, such a configuration is possible. What we will show is that while it is always possible for a vortex to end on a black hole, for small $M$ there is also a phenomenon analogous to flux expulsion: the $X$ field is forced to sit in its unbroken phase $(X=0)$ on the horizon, and the $P$ field takes the form of a monopole potential.

To see this analytically, consider the horizon equations (14a),(14b). These will have the boundary conditions $X$ $=0, P=1$ at $\theta=0$, and $X=X_{m}, P=0$ at $\theta=\pi$. The equation of motion for $P$, Eq. (14b), can be integrated to show that

$$
\lambda(1+\cos \theta)<2 P<(1+\cos \theta)
$$

where $\lambda=1-M^{2} X_{m}^{2} / \beta$. This shows that as $M^{2} / \beta \rightarrow 0, P$ approaches its monopole form, $P_{\text {mon }}=\frac{1}{2}(1+\cos \theta)$. From now on, we will assume that $M^{2} / \beta, M^{2} / N^{2} \ll 1$ and explore the possible solutions for $X$. First of all, note that $X \equiv 0, P$ $\equiv P_{\text {mon }}$ is always a solution to the horizon equations, and it is this that we will call the expulsion solution. Now suppose that a "piercing"' solution exists, then Eq. (14a) implies that $X$ has a local maximum at $\pi$, which we will denote $X_{m}$. Defining $\theta_{0}$ by $\partial_{\theta}\left(\sin \theta X_{, \theta}\right)=0$, we see that at $\theta_{0}, P_{0}^{2}$ $=\left(M^{2} / 2 N^{2}\right) \sin ^{2} \theta_{0}\left(1-X_{0}^{2}\right)$. Since $M / N$ is assumed small, $\theta_{0}$ will obviously be close to $\pi$, and using the bounds on $P$, it is easy to see that

$$
\frac{2}{\pi}\left(\pi-\theta_{0}\right)<\sin \theta_{0}<\frac{4 M}{\sqrt{2} N \lambda}
$$

and so $P$ will be extremely small. Integrating Eq. (14a) between 0 and $\pi$ then gives

$$
\begin{aligned}
\int_{\theta_{0}}^{\pi} X\left[\frac{M^{2}}{2 N^{2}} \sin \theta\left(1-X^{2}\right)-\frac{P^{2}}{\sin \theta}\right] \\
=\int_{0}^{\theta_{0}} X\left[\frac{P^{2}}{\sin \theta}-\frac{M^{2}}{2 N^{2}} \sin \theta\left(1-X^{2}\right)\right] .
\end{aligned}
$$

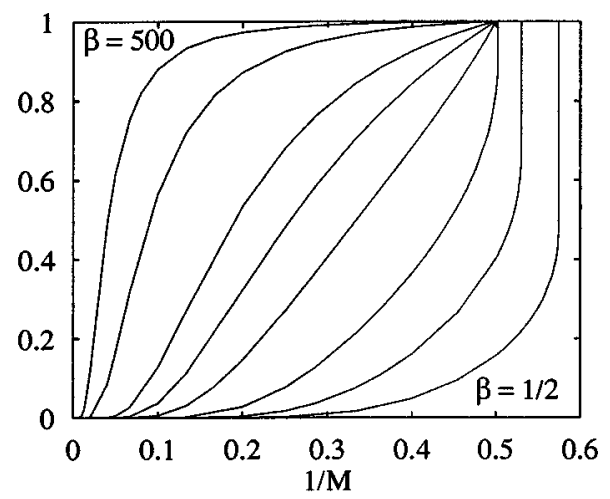

FIG. 6. Values of $X(\pi / 2)$ (left) and $P(\pi / 2)$ as functions of the inverse energy of the extremal black hole. This is done for $N=1$ and the following values of $\beta$ : 500,100,20,10,5,2,1 and $1 / 2$. 

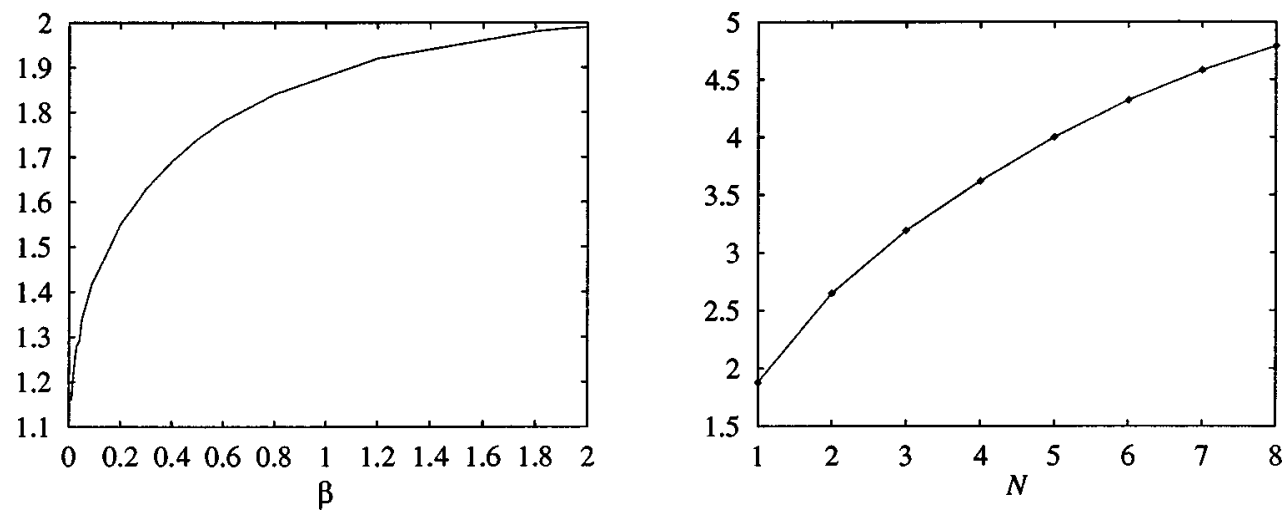

FIG. 7. Values of the "critical mass" $M_{c}$ at which the only solution on the horizon corresponds to the string being expelled, as a function of $\beta$ (for $N=1$ ) and $N$ (for $\beta=1$ ).

The LHS of this equation can readily be bounded above using Eq. (29):

$$
\int_{\theta_{0}}^{\pi} X\left[\frac{M^{2}}{2 N^{2}} \sin \theta\left(1-X^{2}\right)-\frac{P^{2}}{\sin \theta}\right]<\frac{4 M^{4} X_{m}}{N^{4} \lambda^{2}} .
$$

The RHS is a little more tricky to bound below, but noting that $X_{, \theta \theta}$ is positive on $\left[\pi / 2, \theta_{0}\right]$, yet negative $\left[X_{, \theta \theta}\right.$ $\left.=-M^{2} X_{m}\left(1-X_{m}^{2}\right) / 4\right]$ at $\pi$, we can bound $X_{, \theta}$ on $[\pi / 2, \pi]$ by

$$
X_{, \theta}<X_{m} N^{2} \sin ^{3} \theta_{0}
$$

and hence we can bound $X$ below on $(\pi / 2, \pi)$ by

$$
X(\theta)>X_{m}\left[1-\frac{4 \pi M^{3}}{N \lambda^{3}}\right]
$$

therefore

$$
\begin{gathered}
\int_{0}^{\theta_{0}} X\left[\frac{P^{2}}{\sin \theta}-\frac{M^{2}}{2 N^{2}} \sin \theta\left(1-X^{2}\right)\right] \\
>\int_{\pi / 2}^{\theta_{0}} X\left[\frac{P^{2}}{\sin \theta}-\frac{M^{2} \sin \theta}{2 N^{2}}\right] \\
>X_{m} \frac{\lambda^{2}}{16}\left[1-\frac{4 \pi M^{3}}{N \lambda^{3}}\right]\left(1-\frac{8 M^{2}}{N^{2} \lambda^{2}}\right)^{2} .
\end{gathered}
$$

Comparing these bounds on the RHS and LHS of Eq. (30) we see that an expulsion solution is the only possible solution for

$$
M^{4}<\frac{N^{4} \lambda^{4}}{64}\left(1-\frac{4 \pi M^{3}}{N \lambda^{3}}\right)\left(1-\frac{8 M^{2}}{N^{2} \lambda^{2}}\right)^{2} .
$$

For $N=\beta=1$ this gives $M<0.3$. As before, this is a very weak bound, however, the important thing is that it shows that there is a lower bound on the values of $M$ for which the $X$-field can vary on the horizon. For $M$ sufficiently small, the Higgs field is forced to lie in its symmetric phase on the horizon, and we get an expulsion solution.
On the horizon, the single string case differs from the one we have considered previously by the boundary conditions only. At $\theta=0$, we must clearly have a string, but at $\theta=\pi$ nothing forces the fields to assume a vacuum configuration. In fact, we have found that the only smooth solutions were such that $X$ had a vanishing $\theta$-derivative at the South pole. As Fig. 8 shows, the value of $X_{m}$ then depends on the black hole's mass, which means that if we wish to integrate the equations on the whole grid, we also have to update the $\theta$ $=\pi$ boundary. To find equations of motion on this line, we assumed that $P / \sin \theta \rightarrow 0$ and that $X_{, \theta} \rightarrow 0$. The resulting equations, however, were particularly unstable against numerical errors. We were finally able to tackle this problem by artificially coupling the horizon to the rest of the grid (assuming continuity) for some hundred iterations, and by updating the fields at $\theta=\pi$ assuming $X_{, \theta}=P_{, \theta}=0$ there. Examples of a thin string solution piercing the horizon and of a thicker string being expelled are shown on Figs. 9 and 10. Figure 8 shows the variation of the fields on the horizon as we thicken the string, illustrating that for $M=1$ the Higgs field is already expelled from the horizon.

\section{GRAVITATIONAL BACK REACTION}

We begin this section with a lightning review of a selfgravitating cosmic string. For convenience, we will take $N$ $=1$ in this section.

We can readily extend the vortex to a self-gravitating system by using Thorne's cylindrically symmetric coordinate system [21]

$$
d s^{2}=e^{2 \psi} d t^{2}-e^{2(\gamma-\psi)}\left(d z^{2}+d R^{2}\right)-\alpha^{2} e^{-2 \psi} d \phi^{2}
$$

where $\gamma, \psi, \alpha$ are all functions of $R$ only.

In these coordinates, the energy momentum tensor becomes

$$
\begin{aligned}
T_{0}^{0}= & \mathcal{E}=e^{-2(\gamma-\psi)} X^{\prime 2}+\frac{e^{2 \psi} X^{2} P^{2}}{\alpha^{2}} \\
& +\beta e^{2(\gamma-2 \psi)} \frac{P^{\prime 2}}{\alpha^{2}}+\frac{1}{4}\left(X^{2}-1\right)^{2}
\end{aligned}
$$



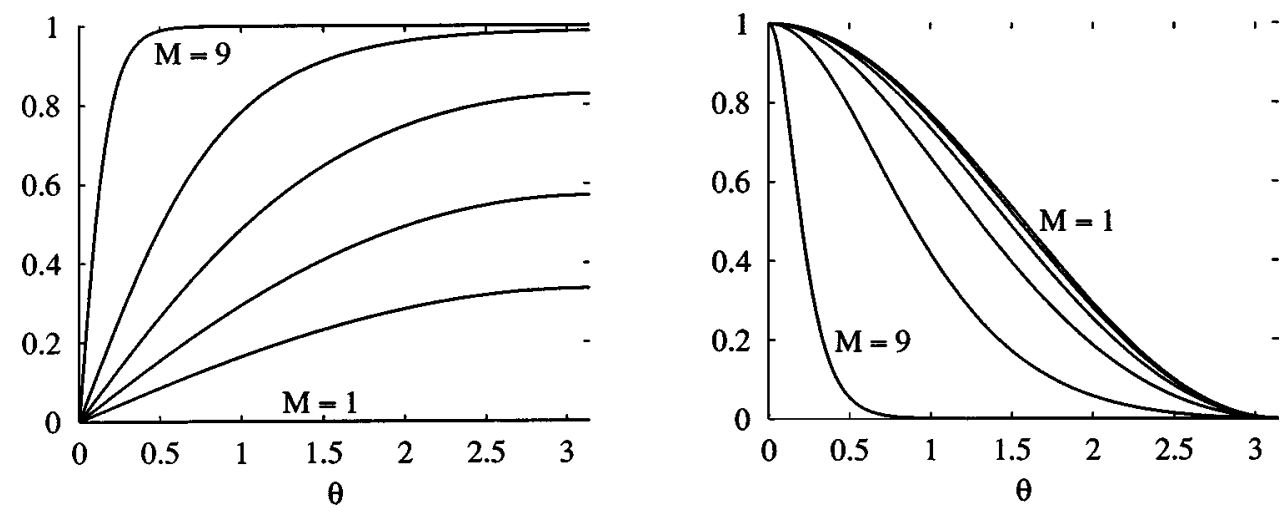

FIG. 8. Fields $X(\theta)$ (left) and $P(\theta)$ on the horizon for the case of a single string ending on the black hole. The string has a Higgs width of 1 , and we plot the profiles for $\beta=N=1$ and the following values of $M: 9,2,1.3,1.1,1.03,1$.

$$
\begin{aligned}
T_{R}^{R}= & -\mathcal{P}_{R}=-e^{-2(\gamma-\psi)} X^{\prime 2}+\frac{e^{2 \psi} X^{2} P^{2}}{\alpha^{2}}-\beta e^{2(\gamma-2 \psi)} \frac{P^{\prime 2}}{\alpha^{2}} \\
& +\frac{1}{4}\left(X^{2}-1\right)^{2} \\
T_{\phi}^{\phi}= & -\mathcal{P}_{\phi}=e^{-2(\gamma-\psi)} X^{\prime 2}-\frac{e^{2 \psi} X^{2} P^{2}}{\alpha^{2}} \\
& -\beta e^{2(\gamma-2 \psi)} \frac{P^{\prime 2}}{\alpha^{2}}+\frac{1}{4}\left(X^{2}-1\right)^{2} \\
T_{z}^{z}= & -\mathcal{P}_{z}=T_{0}^{0} .
\end{aligned}
$$

To zeroth order in $\epsilon=8 \pi G \eta^{2}$ introduced in Sec. II

$$
\alpha=R, \quad \psi=\gamma=0, \quad X=X_{0}, \quad P=P_{0},
$$

and conservation of the energy-momentum tensor gives

$$
\left(R \mathcal{P}_{0^{R}}\right)^{\prime}=\mathcal{P}_{0} \phi
$$

To first order in $\epsilon$ the string metric is given by [22]

$$
\begin{aligned}
& \alpha=\left[1-\epsilon \int_{0}^{R} R\left(\mathcal{E}_{0}-\mathcal{P}_{0^{R}}\right) d R\right] R+\epsilon \int_{0}^{R} R^{2}\left(\mathcal{E}_{0}-\mathcal{P}_{0}\right) d R \\
& \gamma=2 \psi=\epsilon \int_{0}^{R} R \mathcal{P}_{0^{R}} d R
\end{aligned}
$$

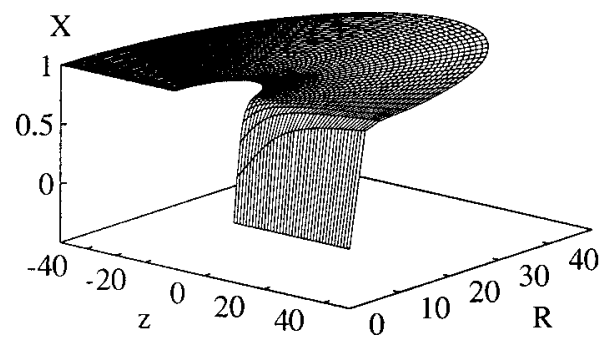

where the subscript zero indicates evaluation in the flat space limit. Since the string functions $X$ and $P$ rapidly fall off to their vacuum values outside the core, the integrals in Eq. (40) rapidly converge to their asymptotic, constant, values. Writing

$$
\begin{aligned}
& A=\int_{0}^{\infty} R\left(\mathcal{E}_{0}-\mathcal{P}_{0^{R}}\right) d R, \\
& B=\int_{0}^{\infty} R^{2}\left(\mathcal{E}_{0}-\mathcal{P}_{0^{R}}\right) d R \quad \text { and } \quad C=\int_{0}^{\infty} R \mathcal{P}_{0} R,
\end{aligned}
$$

then the asymptotic form of the metric is

$$
\begin{aligned}
d s^{2} & =e^{\epsilon C}\left[d t^{2}-d R^{2}-d z^{2}\right]-R^{2}(1-\epsilon A)^{2} e^{-\epsilon C} d \phi^{2} \\
& =d \hat{t}^{2}-d \hat{R}^{2}-d \hat{z}^{2}-\hat{R}^{2}(1-\epsilon(A+C))^{2} d \phi^{2},
\end{aligned}
$$

where $\hat{t}=e^{\epsilon C / 2} t$, etc. This is a conical metric with deficit angle

$$
\Delta=2 \pi \epsilon(A+C)=2 \pi \epsilon \int R \mathcal{E}_{0} d R=8 \pi G \mu,
$$

where $\mu$ is the energy per unit length of the string. Notice that the deficit angle is independent of the radial stresses, but that when the radial stresses do not vanish there is a red/blueshift of time between infinity and the core of the string. The only case in which these stresses do vanish is when $\beta=1$.

Now we are ready to consider the gravitational effect of the string superimposed on the black hole. For this we need

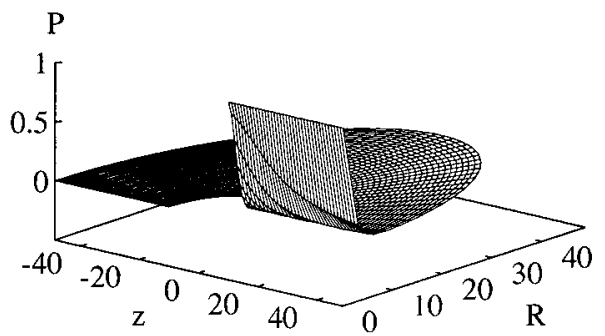

FIG. 9. Solution $X(r, \theta)$ and $P(r, \theta)$ for a single string ending on the black hole, and $M=Q=10, \beta=N=1, \varepsilon=10^{-4}, N_{r}=N_{\theta}=50$. 

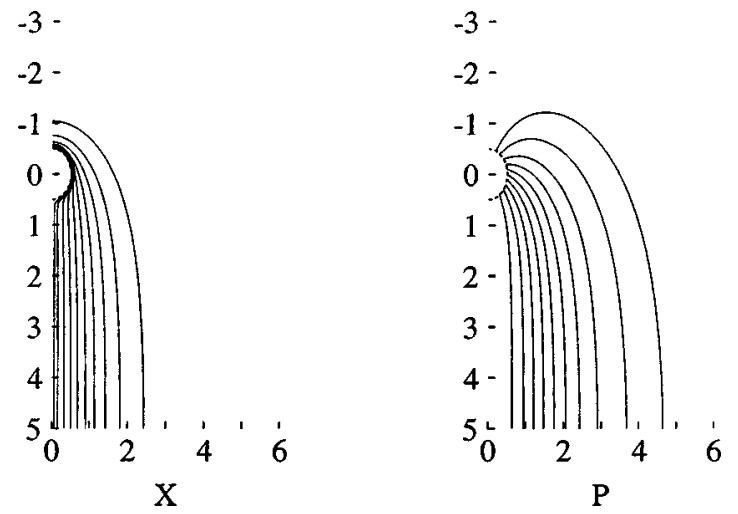

FIG. 10. Contours of $X$ and $P$ for a single string ending on the black hole, and $M=Q=1 / 2, \beta=N=1, N_{r}=N_{\theta}=100, r_{m}=10$.

to consider a general static axially symmetric solution to the Einstein-Maxwell-Abelian Higgs equations derived from the action $S_{1}+S_{2}$, where $S_{1}$ is given by Eq. (1), and $S_{2}$ is the Einstein-Maxwell action

$$
S_{2}=-\frac{1}{16 \pi G} \int d^{4} x \sqrt{-g}\left[R+F_{\mu \nu}^{2}\right]
$$

where for clarity in what follows, we have rescaled the electromagnetic field by $\sqrt{G}$. We may pick coordinates so that the metric takes the form

$$
d s^{2}=e^{2 \psi} d t^{2}-e^{2(\gamma-\psi)}\left(d z^{2}+d \rho^{2}\right)-\alpha^{2} e^{-2 \psi} d \phi^{2},
$$

where $\psi, \gamma, \alpha$ are independent of $t, \phi$. [Note the deliberate similarities with the Thorne metric (36).] We then apply an iterative procedure to solving the field equations in the thin string limit, starting with the Reissner-Nordstrom background solution, the Nielsen-Olesen forms of $X$ and $P$, and expanding the equations of motion in terms of $\epsilon=8 \pi G \eta^{2}$ as before.

The usual Reissner-Nordstrøm metric (7) is of course written in "spherical" coordinates, whereas, in such an iterative process we require it in axisymmetric coordinates. For future reference the coordinate transformation is

$$
\rho=\sqrt{r^{2}-2 M r+Q^{2}} \sin \theta, \quad z=(r-M) \cos \theta
$$

and the metric and (rescaled) electromagnetic gauge potential (in a suitable gauge) are given by

$$
\begin{aligned}
d s^{2}= & \frac{\left(R_{+}+R_{-}\right)^{2}-4 \Delta^{2}}{\left(R_{+}+R_{-}+2 M\right)^{2}} d t^{2} \\
& -\frac{\left(R_{+}+R_{-}+2 M\right)^{2}}{4 R_{+} R_{-}}\left(d \rho^{2}+d z^{2}\right) \\
& -\rho^{2} \frac{\left(R_{+}+R_{-}+2 M\right)^{2}}{\left(R_{+}+R_{-}\right)^{2}-4 \Delta^{2}} d \phi^{2},
\end{aligned}
$$

$$
A_{0 \nu}= \begin{cases}\frac{2 Q z}{R_{+}+R_{-}} \partial_{\nu} \phi & \text { magnetic } \\ \frac{2 Q}{\left(R_{+}+R_{-}+2 M\right)} \partial_{\nu} t & \text { electric, }\end{cases}
$$

where

$$
R_{ \pm}=(z \pm \Delta)^{2}+\rho^{2}
$$

and $\Delta^{2}=M^{2}-Q^{2}$.

Returning to the general system, the relevant equations of motion are

$$
\begin{gathered}
0=\partial_{\rho}\left(\alpha F_{\rho}^{\nu}\right)+\partial_{z}\left(\alpha F_{z}^{\nu}\right) \\
\alpha_{, z z}+\alpha_{, \rho \rho}=-\sqrt{-g}\left(\mathcal{T}_{z}^{z}+\mathcal{T}_{\rho}^{\rho}\right) \\
\left(\alpha \psi_{, z}\right)_{, z}+\left(\alpha \psi_{, \rho}\right)_{, \rho}=\frac{1}{2} \sqrt{-g}\left(\mathcal{T}_{0}^{0}-\mathcal{T}_{z}^{z}-\mathcal{T}_{\rho}^{\rho}-\mathcal{T}_{\phi}^{\phi}\right) \\
\gamma_{, \rho \rho}+\gamma_{, z z}=-\psi_{, \rho}^{2}-\psi_{, z}^{2}-e^{2(\gamma-\psi)} \mathcal{T}_{\phi}^{\phi},
\end{gathered}
$$

where the energy momentum tensor is given by

$$
\mathcal{T}_{b}^{a}=E_{b}^{a}+\epsilon T_{b}^{a}
$$

The first term is the electromagnetic contribution to the stress-energy which is given by

$$
E_{\nu}^{\mu}=-F_{\nu \lambda} F^{\mu \lambda}+\frac{1}{4} F^{2} \delta_{\nu}^{\mu}
$$

and the last term is the contribution from the string, which has the explicit form

$$
\begin{aligned}
T_{0}^{0}= & V(X)+\frac{X^{2} P^{2}}{\alpha^{2} e^{-2 \psi}}+\left[\frac{\left(P_{, \rho}^{2}+P_{, z}^{2}\right)}{\beta^{-1} \alpha^{2} e^{-2 \psi}}\right. \\
& \left.+\left(X_{, \rho}^{2}+X_{, z}^{2}\right)\right] e^{-2(\gamma-\psi)} \\
T_{\phi}^{\phi}= & V(X)-\frac{X^{2} P^{2}}{\alpha^{2} e^{-2 \psi}}+\left[-\frac{\left(P_{, \rho}^{2}+P_{, z}^{2}\right)}{\beta^{-1} \alpha^{2} e^{-2 \psi}}\right. \\
& \left.+\left(X_{, \rho}^{2}+X_{, z}^{2}\right)\right] e^{-2(\gamma-\psi)} \\
T_{\rho}^{\rho}+T_{z}^{z}= & 2 V(X)+\frac{2 X^{2} P^{2}}{\alpha^{2} e^{-2 \psi}} .
\end{aligned}
$$

Note that the electromagnetic stress energy always satisfies $E_{\rho}^{\rho}+E_{z}^{z}=0$.

As in [3] we now write $\alpha=\alpha_{0}+\epsilon \alpha_{1}$ etc., and solve the Einstein-Maxwell and the string equations iteratively. The main difference to [3] is that we now have the electromagnetic gauge potential present, which appears at $\mathrm{O}\left(\epsilon^{0}\right)$. This 
means that to $\mathrm{O}(\epsilon)$ the geometry is not only affected by the string, but also by the back reaction of the string on the electromagnetic field.

To zeroth order, we have the background solutions (47), (48), $X=X_{0}(R)$ and $P=P_{0}(R)$ where $R=r \sin \theta=\rho e^{-\psi_{0}}$ as before. Using the coordinate transformation (46), one finds that

$$
R_{, z}^{2}+R_{, \rho}^{2}=\frac{r^{2}}{R_{+} R_{-}}\left(1-\frac{2 M}{r} \sin ^{2} \theta+\frac{Q^{2}}{r^{2}} \sin ^{2} \theta\right) \simeq e^{2\left(\gamma_{0}-\psi_{0}\right)}
$$

in the core of the string, where $\sin \theta=O\left(M^{-1}\right)$. Therefore, in and near the core of the string, the relevant combinations of the zeroth order energy momentum tensor of the string are

$$
\begin{gathered}
\left(T_{0}\right)^{0}{ }_{0}=\mathcal{E}+O\left(M^{-2}\right) \\
\left(T_{0}\right)^{\phi}{ }_{\phi}=-\mathcal{P}_{\phi}+O\left(M^{-2}\right) \\
\left(T_{0}\right)_{\rho}^{\rho}+\left(T_{0}\right)_{z}^{z}=-\left(\mathcal{P}_{R}+\mathcal{P}_{\phi}\right) .
\end{gathered}
$$

As in [3] we will assume (and show subsequently that it is consistent to do so) that the perturbed solutions take the form

$$
\alpha_{1}=\rho a(R), \quad \psi_{1}=\psi_{1}(R), \quad \gamma_{1}=\gamma_{1}(R), \quad A_{1} \bar{\mu}=f(R) A_{0} \mu
$$

Computing the necessary derivative of $R$ gives from Eq. (50b) the following equation for $a(R)$ :

$$
\begin{aligned}
{[1-} & \left.\frac{R^{2}}{r^{2}}\left(\frac{2 M}{r}-\frac{Q^{2}}{r^{2}}\right)\right] a^{\prime \prime}(R) \\
& +\left[\frac{2}{R}-\frac{Q^{2}}{2 r^{4}}-\frac{R}{r^{2}}\left(\frac{3 M}{r}-\frac{Q^{2}}{r^{2}}\right)\right] a^{\prime}(R) \\
& =\frac{1}{R^{2}}\left(R^{2} a^{\prime}\right)^{\prime}+O\left(M^{-2}\right)=-\left(\mathcal{E}-\mathcal{P}_{R}\right)+O\left(M^{-2}\right),
\end{aligned}
$$

which is consistently solved, as in the Schwarzschild case, by

$$
\begin{aligned}
a(R)= & -\int R\left[\mathcal{E}_{0}-\mathcal{P}_{0 R}\right] d R \\
& +\frac{1}{R} \int R^{2}\left[\mathcal{E}_{0}-\mathcal{P}_{0 R}\right] d R \\
& \sim-A+\frac{B}{R} \quad \text { as } R \rightarrow \infty .
\end{aligned}
$$

Now look at the Maxwell equation for an electric potential (the magnetic potential can be obtained by a duality transformation). Substituting in the assumed form for the functions (56), we obtain, for $f(R)$,

$$
\frac{f^{\prime \prime}(R)}{R}+\frac{f^{\prime}(R)}{R^{2}}=\frac{\rho^{2}}{r^{2} R^{2}}\left(a^{\prime}-2 \psi_{1}^{\prime}\right)=O\left(M^{-2}\right),
$$

which implies that $f=f_{0}$, a constant. Turning to the $\psi$ equation, and inputting in this form of $f$, we find

$$
\begin{aligned}
\psi_{1}^{\prime \prime}[ & \left.1+\frac{R^{2}}{r^{2}}\left(\frac{Q^{2}}{r^{2}}-\frac{2 M}{r}\right)\right]+\frac{\psi_{1}^{\prime}}{R}\left[1-\frac{R^{2} Q^{2}}{r^{4}}\right]+\frac{a^{\prime} R}{r^{2}}\left(\frac{Q^{2}}{r^{2}}+\frac{M}{r}\right) \\
& =\frac{1}{2}\left(\mathcal{P}_{R}+\mathcal{P}_{\phi}\right)+\frac{Q^{2}}{r^{4}}\left[2 f_{0}-2 \psi_{1}\right] .
\end{aligned}
$$

This is solved by

$$
\psi_{1}=\frac{1}{2} \int R \mathcal{P}_{R} \sim \frac{C}{2} \quad \text { as } R \rightarrow \infty, \quad f_{0}=C / 2
$$

the latter value of $f_{0}$ being set by consistency of the $\psi$ equation outside the core. It is then straightforward to check that $\gamma_{1}=2 \psi_{1}$. The magnetic correction is obtained either directly, or via duality, to be

$$
f_{M}(R)=a(R)-2 \psi_{1}(R)+C / 2 \text {. }
$$

As in [3], the corrections are almost the same as for the self-gravitating cosmic string. After transforming back to Schwarzschild coordinates, the metric outside the string core becomes

$$
\begin{aligned}
d s^{2}= & e^{\epsilon C}\left[\left(1-\frac{2 M}{r}+\frac{Q^{2}}{r^{2}}\right) d t^{2}-\frac{d r^{2}}{1-\frac{2 M}{r}+\frac{Q^{2}}{r^{2}}}-r^{2} d \theta^{2}\right] \\
& -r^{2} \sin ^{2} \theta(1-\epsilon A)^{2} e^{-\epsilon C} d \phi^{2}
\end{aligned}
$$

where we have neglected the $B$ term from $a(R)$ since it yields a correction $O(G \mu) \times O\left(E^{-1}\right)$. The gauge potentials are

$$
A_{\nu}= \begin{cases}Q(1-\cos \theta)[1-\epsilon(A+C / 2)] \partial_{\nu} \phi & \text { magnetic, } \\ -\frac{Q}{r}[1+\epsilon C / 2] \partial_{\nu} t & \text { electric. }\end{cases}
$$

If we now rescale the metric so that $\hat{t}=e^{\epsilon C / 2} t$, etc. (and, accordingly, rescale the parameters $M$ and $Q$ ) we find

$$
\begin{aligned}
d s^{2}= & \left(1-\frac{2 \hat{M}}{\hat{r}}+\frac{\hat{Q}^{2}}{\hat{r}^{2}}\right) d \hat{t}^{2}-\frac{d \hat{r}^{2}}{1-\frac{2 \hat{M}}{\hat{r}}+\frac{\hat{Q}^{2}}{\hat{r}^{2}}}-\hat{r}^{2} d \theta^{2} \\
& -\hat{r}^{2} \sin ^{2} \theta(1-\epsilon A)^{2} e^{-2 \epsilon C} d \phi^{2} .
\end{aligned}
$$

Again, we find a deficit angle $\Delta=2 \pi \epsilon(A+C)=8 \pi G \mu$. Besides, the gravitational mass of the black hole, $M_{g}$, which is given by the coefficient of $2 \hat{r}^{-1}$ in $g_{\hat{t}} \hat{t}$, has been shifted in the presence of the string to $M_{g}=\hat{M}=e^{\epsilon C / 2} M$. 
On the other hand, the internal energy of the black hole (its ADM mass, appropriately generalized to asymptotically locally flat spaces, see e.g., [23]) is now

$$
M_{I}=\hat{M}(1-\epsilon A) e^{-\epsilon C}=M(1-\epsilon A) e^{-\epsilon C / 2} .
$$

Not only is the mass of the black hole corrected, but the physical charge of the black hole, defined as

$$
Q_{p h}= \begin{cases}\frac{1}{4 \pi} \int_{S^{2}} F & \text { magnetic, } \\ \frac{1}{4 \pi} \int_{S^{2}} * F & \text { electric, }\end{cases}
$$

becomes

$$
Q_{p h}=Q[1-\epsilon(A+C / 2)] .
$$

Notice that $M_{g} / Q_{p h}>M_{I} / Q_{p h}=M / Q$.

We can now write the first-order corrected solution in terms of the physical parameters $M_{I}, Q_{p h}, \mu$, as

$$
\begin{aligned}
d s^{2}= & \left(1-e^{4 G \mu} \frac{2 M_{I}}{\hat{r}}+e^{8 G \mu} \frac{Q_{p h}^{2}}{\hat{r}^{2}}\right) d \hat{t}^{2} \\
& -\frac{d \hat{r}^{2}}{1-e^{4 G \mu} \frac{2 M_{I}}{\hat{r}}+e^{8 G \mu} \frac{Q_{p h}^{2}}{\hat{r}^{2}}} \\
& -\hat{r}^{2} d \theta^{2}-e^{-4 G \mu} \hat{r}^{2} \sin ^{2} \theta d \phi^{2},
\end{aligned}
$$

and

$$
A_{\nu}= \begin{cases}Q_{p h}(1-\cos \theta) \partial_{\nu} \phi & \text { magnetic, } \\ -e^{4 G \mu} \frac{Q_{p h}}{\hat{r}} \partial_{\nu} \hat{t} & \text { electric. }\end{cases}
$$

The corrected inner and outer horizons exterior to the core are therefore at

$$
\hat{r}_{ \pm}=e^{4 G \mu}\left(M_{I} \pm \sqrt{M_{I}^{2}-Q_{p h}^{2}}\right),
$$

since we are assuming that the string is thin with respect to the back hole, these expressions will hold except at the poles of the horizon. Notice that the condition that the black hole is extremal (i.e., its horizon is degenerate), is that $M_{I}=Q_{p h}$. Finally, we can use the methods of [23] to find the entropy using the Bekenstein-Hawking formula

$$
S=\frac{\mathcal{A}_{h}}{4 G}=16 \pi e^{4 G \mu} \hat{r}_{+}^{2} .
$$

Consider now an extremal black hole-cosmic string merger. If we keep the internal energy and the charge of the black hole fixed (i.e., impose microcanonical boundary conditions), then we find, as in [3], that the change in the gravitational mass, $\delta M_{g}=4 G \mu M_{g}$ equals the energy of the length of string swallowed by the black hole. Since the en- tropy increases, we see that merging is thermodynamically favored. And more remarkably, we see that, at least to first order, the black hole remains extremal after the merger.

\section{DISCUSSION}

Our analysis in this paper appears to settle the question of whether or not a vortex can penetrate an extremal black hole. We have provided analytical proofs that vortices of size smaller than a certain fraction (of order unity) of the radius of the black hole will definitely pierce the horizon, whereas vortices thicker than a certain lower bound will instead wrap the black hole. The numerical analysis confirms this, and, for $N=\beta=1$, places the transition at $M=1.8865$, or $G m$ $=1.8865 / \sqrt{\lambda} \eta$. For a single string ending on the black hole the Higgs field presents a similar behavior, i.e., it vanishes on the horizon only if the black hole is small enough. In that regime, the magnetic field, instead of being expelled, takes the form of a monopole field. Thus we see that single strings are always allowed to end on black holes, which solves one of the paradoxes that the results of [12] seemed to pose. Finally, we have computed the back reaction effect of a thin vortex on the geometry. This results in the expected conical geometry, but we have been able to check as well that the black hole remains extremal after including the corrections to the mass and the charge.

Given that in [19] the expulsion of the (unbroken) magnetic field was related to a sort of "superconducting" behavior of the extremal black hole, one would be tempted to interpret the penetrating solutions as exhibiting the wellknown breakdown of the superconducting state for strong enough magnetic fields. However, this does not seem to be the case here. In [19] exact solutions (which account fully for the back reaction of the gauge field) are presented for extremal black holes in magnetic fields, and the expulsion persists no matter how strong the magnetic fields are taken to be.

As a matter of fact, we can argue that, far from having anything to do with the strength of the magnetic field, it is instead the presence of a mass for the gauge vector which spoils the expulsion from the extremal horizon. In order to illustrate this point, consider a massive vector (Proca) field, with an explicit mass $\mu$. On the extremal horizon the field equation becomes

$$
\sin \theta \partial_{\theta}\left(\frac{\partial_{\theta} P}{\sin \theta}\right)-\mu^{2} P=0 .
$$

This equation does not admit a constant solution (apart from the trivial $P=0)$. Therefore $G_{\theta \phi} \propto \partial_{\theta} P_{\phi} \neq 0$ and we find a (locally) non-vanishing flux of the field across the horizon.

As we have seen, things are subtler when the mass originates from spontaneous breaking of the gauge symmetry. The system has both massive and massless phases, and both expulsion and penetration can be found, depending essentially on the relative values of the vortex and black hole radii. But the argument above shows that the expulsion of the field can take place only if the symmetry is exactly restored on the horizon. It is therefore quite remarkable that, in cer- 
tain regimes, the geometry of the extremal horizon can locally enforce the exact restoration of the symmetry.

The transition from penetration to expulsion can be viewed as a phase transition on the horizon of the black hole. In particular, Fig. 6 is reminiscent of the behavior of, say, the magnetization of a ferromagnet as a function of temperature, or more generally, the order parameter of a system undergoing a second order phase transition. The order parameter is in this case the value of the Higgs field on the horizon, $X$, and instead of a function of the temperature, the phase transition takes place when we vary the (inverse) size of the horizon $\sim M^{-1}$. Beyond a certain critical value, $M^{-1} \geqslant M_{c}^{-1}$, the symmetry is restored throughout the horizon. Notice that the transition takes place when the energy scale set by the black hole, $M$, is similar to the Higgs energy $\sqrt{\lambda} \eta$. In a sense, this would be the natural expectation, since $\eta$ sets the energy scale for symmetry restoration. But it should be stressed that this expectation is only realized for extremal black holes: the symmetry is never restored on non-extremal horizons. ${ }^{1}$ It might be interesting to pursue this analogy further, and study, e.g., critical exponents near the transition point, such as $X \sim\left|M-M_{c}\right|^{\widetilde{\beta}}$ (and see, e.g., how $\widetilde{\beta}$ varies, or not, at different points on the horizon).

Another interesting question that can be explored in more generality is the interaction between the straight cosmic string and the extremal black hole. The set up we have been considering so far places the vortex in perfect alignment with a black hole axis. If the black hole and the vortex are displaced relative to each other, the symmetry of the system decreases and complication increases greatly. We can, however, analyze in some detail the interactions between the extremal black hole and the cosmic string when the string is thin enough to allow us to effectively integrate out the details of the core structure. We can proceed in several levels of approximation. A very crude approximation would be taking the black hole as a test particle in the background of a selfgravitating cosmic string, i.e., in a flat spacetime with a conical defect. As is well known, since the spacetime is locally flat the test particle does not experience any force.

We can improve on this by accounting for the gravitational field of the black hole. In the Newtonian approximation, we would be solving the Poisson equation for the Newtonian potential in a conical spacetime. The effect of the conical defect on a massive neutral particle can be understood by viewing the particle as subject to a force coming from the "images" produced by the conical defect. This results in the neutral particle being attracted towards the string [16] (see also [24]). In contrast, when applied to a charged "extremal particle," such that $m=q$, this argument

\footnotetext{
${ }^{1}$ Notice as well that it would be incorrect to think that the restoration of the symmetry comes about as an effect of the thermal properties of black holes. For one thing, an extremal black hole has zero temperature. Moreover, the thermality is only seen when accounting for quantum effects, whereas here we work at the classical level.
}

would yield a vanishing force, since the gravitational and electrostatic forces between the particle and its images cancel out.

Actually, for extremal black holes this result holds not only in the Newtonian approximation, but also exactly in the full Einstein-Maxwell theory [25]. To see this, recall first that the solution for an (electric) extremal black hole can be written as

$$
\begin{gathered}
d s^{2}=H^{-2} d t^{2}-H^{2}\left(d x^{2}+d y^{2}+d z^{2}\right), \\
A_{\mu}=\left(H^{-1}-1\right) \partial_{\mu} t .
\end{gathered}
$$

Written in this fashion, the Einstein-Maxwell equations only require that $H$ is a harmonic function in the flat $(x, y, z)$ space, $\nabla_{x, y, z}^{2} H=0$. The extremal black hole is recovered by setting

$$
H=1+\frac{Q}{\sqrt{\left(x-x_{0}\right)^{2}+\left(y-y_{0}\right)^{2}+\left(z-z_{0}\right)^{2}}},
$$

and the horizon is at $(x, y, z)=\left(x_{0}, y_{0}, z_{0}\right){ }^{2}$ If we want to include the cosmic string, then we just have to solve the Laplace equation for $H$, this time in a space with a straight conical line. The relevant solution has been given in many places, see e.g., in the context of cosmic strings [16]. In cylindrical coordinates $(\rho, z, \phi)$ centered on the string, with conical deficit such that $0 \leqslant \phi \leqslant 2 \pi / p$, if we put the black hole at $\rho=\rho_{0}, \phi=0$ and $z=0$, then

$$
\begin{aligned}
& H_{p}\left(z, \rho, \phi ; \rho_{0}\right) \\
& =1+\frac{Q}{\pi \sqrt{2 \rho \rho_{0}}} \int_{u_{o}}^{\infty} \frac{d u}{\sqrt{\cosh u-\cosh u_{0}}} \\
& \quad \times \frac{p \sinh p u}{\cosh p u-\cos p \phi},
\end{aligned}
$$

where $u_{0}$ is defined by

$$
\cosh u_{0}=\frac{\rho^{2}+z^{2}+\rho_{0}^{2}}{2 \rho \rho_{0}} .
$$

This solution is nonsingular, away from the conical line and the singularity of the black hole. Thus, there are no forces between the extremal black hole and the cosmic string.

At distances much larger than $\rho_{0}$, the harmonic function becomes

$$
H_{p} \rightarrow 1+\frac{Q p}{\sqrt{\rho^{2}+z^{2}}}
$$

Since $p \approx 1+4 G \mu$, we see that we reproduce the gravitational and electric potentials in Eqs. (69), (70), with the parameter $Q$ equaling both the internal energy $M_{I}$ and the

\footnotetext{
${ }^{2}$ The "Schwarzschild coordinates" used in Eq. (12) correspond to setting $r-M=\left[\left(x-x_{0}\right)^{2}+\left(y-y_{0}\right)^{2}+\left(z-z_{0}\right)^{2}\right]^{1 / 2}$.
} 
physical charge $Q_{p h}$ we had introduced earlier. The result, however, is independent of whether the string and the black hole are merged or not. Whenever the black hole is in the presence of a cosmic string, the lines of force are "focused," resulting in an increase of the gravitational and electric potentials at long distances.

The same conclusion regarding the absence of a force between the two objects can be reached again from another perspective. This time, neglect the gravitational back reaction of the string, and consider a Nambu-Goto string in the background of the extremal black hole, with metric as in Eq. (74). The static interaction potential $V(x, y, z)$ experienced by a string can be read off from the string action, $I_{N G}$ $\sim \int d \tau V(x, y, z)$. If we take a straight string along, say, the $z$ axis, this is, $T=\tau, Z=\sigma, X, Y=$ const, then the action is

$$
I_{N G}=-\mu \int d \tau d \sigma \sqrt{-\operatorname{det} g_{\mu \nu} \partial_{\alpha} X^{\mu} \partial_{\beta} X^{\nu}}=-\mu L \int d \tau,
$$

( $L$ is the length of the string) i.e., the static force vanishes. With a bit more work it is easy to see that if the string is given a velocity transverse to its axis, then its motion is slowed down as it approaches the black hole.

Clearly, in all these arguments we have been neglecting the effect of the black hole on the string core. But nevertheless we seem to find that, at least if they are well separated, a straight string and an extremal black hole hardly feel each other's presence.

These results appear to imply that the binding energy between the infinitely thin string and the extremal black hole is zero. It appears difficult to make a clear comparison between the energy of a finite-radius vortex before and after the merger. On the one hand, when the vortex penetrates the horizon, a hole is cut out from space, where a part of the string is missing. As we have seen in the previous section, the energy of the missing link equals the change in the gravitational mass of the black hole. On the other hand, since the geometry along the string changes in the process, one should be careful about how to define the total energy change. In particular, since the string has an infinite length (and hence, infinite energy), one should specify a regularization, and choose how to fix a large but finite length of the string before and after the merger.

We conclude by mentioning that it should be interesting to include fermion fields and study the supersymmetry of the extreme black hole-vortex configuration. As is well known, in the absence of the cosmic string, the Reissner-Nordstrom black hole can be embedded in $(N=2)$ supergravity, and the extremality condition $M=Q$ appears then as the BPS condition for the existence of unbroken supersymmetry generators [26]. Similarly, the Nielsen-Olesen vortex with $\beta=1$ admits a supersymmetric embedding in $\mathrm{N}=2$ supergravity in three dimensions [27], and the solution preserves half of the Killing spinors of the flat vacuum. A natural question to ask is whether the merger configuration will be supersymmetric as well. Although we have not analyzed this point in any detail, our analysis indicates that when the vortices are infinitely thin, the system exhibits some of the features characteristic of Bogomol'nyi-Prasad-Sommerfield (BPS) configurations, including the equality of the ADM mass and the physical charge, $M_{I}=Q_{p h}$ (which was actually obtained for finite, if small, width strings), as well as the absence of forces and vanishing binding energy between the two objects. ${ }^{3}$ Even when the vortices have a finite width, we suspect that it is also possible to maintain another feature of BPS systems, namely the reduction of the equations of motion to a first order system. Although at first sight this seems unlikely given the lack of symmetry, it was shown in Anderson et al. [28] that for a general world sheet embedding, such a reduction to a first order system does occur. In this case we have a curved geometry, nonetheless, preliminary indications are that a generalization of this method will work. However, the drastic change in the behavior that takes place as the vortex grows thicker (from penetration to expulsion) would make it very surprising that supersymmetry be present in general.

\section{ACKNOWLEDGMENTS}

We would like to thank Ana Achúcarro, Andrew Chamblin, Konrad Kuijken, and Andrew Sornborger for conversations, and particularly K.K. and A.S. for making their programs freely available for comparison. F.B. is supported by the ORS, the Swiss National Science Foundation and Durham University; R.E. is supported by EPSRC; and R.G. is supported by the Royal Society.

\footnotetext{
${ }^{3}$ The fact that entropy is generated in the merger is known to happen as well in BPS composite black holes, and is typically accompanied by a reduction of the number of supersymmetry generators that are preserved.
}

[1] R. Bartnik and J. McKinnon, Phys. Rev. Lett. 61, 141 (1988).

[2] K. Lee, V.P. Nair, and E.J. Weinberg, Phys. Rev. Lett. 68, 1100 (1992); Phys. Rev. D 45, 2751 (1992).

[3] A. Achúcarro, R. Gregory, and K. Kuijken, Phys. Rev. D 52, 5729 (1995).

[4] D. Eardley, G. Horowitz, D. Kastor, and J. Traschen, Phys. Rev. Lett. 75, 3390 (1995).

[5] S.W. Hawking and S.F. Ross, Phys. Rev. Lett. 75, 3382
(1995).

[6] R. Emparan, Phys. Rev. Lett. 75, 3386 (1995).

[7] R. Gregory and M. Hindmarsh, Phys. Rev. D 52, 5598 (1995).

[8] R. Emparan, Phys. Rev. D 52, 6976 (1995).

[9] H.B. Nielsen and P. Olesen, Nucl. Phys. B61, 45 (1973).

[10] M. Aryal, L. Ford and A. Vilenkin, Phys. Rev. D 34, 2263 (1986).

[11] R. Gleiser and J. Pullin, Class. Quantum Grav. 6, L141 (1989). 
[12] A. Chamblin, J.M.A. Ashbourn-Chamblin, R. Emparan, and A. Sornborger, Phys. Rev. D 58, 124014 (1998); Phys. Rev. Lett. 80, 4378 (1998).

[13] F. Bonjour and R. Gregory, Phys. Rev. Lett. 81, 5034 (1998).

[14] A. Achúcarro and R. Gregory, Phys. Rev. Lett. 79, 1972 (1997).

[15] B. Linet, Phys. Rev. D 33, 1833 (1986); B. Boisseau, C. Charmousis, and B. Linet, Class. Quantum Grav. 13, 1797 (1996).

[16] A.G. Smith, in The Formation and Evolution of Cosmic Strings, edited by G.W. Gibbons, S.W. Hawking, and T. Vachaspati (Cambridge University Press, Cambridge, England, 1990), p. 263.

[17] E.B. Bogomolnyi, Yad. Fiz. 24, 861 (1976) [Sov. J. Nucl. Phys. 24, 449 (1976)].

[18] A. Achúcarro, R. Gregory, J. Harvey, and K. Kuijken, Phys. Rev. Lett. 72, 3646 (1994).

[19] A. Chamblin, R. Emparan, and G.W. Gibbons, Phys. Rev. D 58, 084009 (1998).
[20] William H. Press, Saul A. Teukolsky, William T. Vetterling, and Brian P. Flannery, Numerical Recipes in C. The Art of Scientific Computing, 2nd ed. (Cambridge University Press, Cambridge, England, 1992).

[21] K.S. Thorne, Phys. Rev. 138, 251 (1965).

[22] R. Gregory, Phys. Rev. Lett. 59, 740 (1987); D. Garfinkle, Phys. Rev. D 32, 1323 (1985).

[23] S.W. Hawking and G.T. Horowitz, Class. Quantum Grav. 13, 1487 (1996).

[24] W.B. Perkins and A.C. Davis, Nucl. Phys. B349, 207 (1991); B. Allen, J.G. McLaughlin, and A.C. Ottewill, Phys. Rev. D 45, 4486 (1992).

[25] B. Linet, Class. Quantum Grav. 4, L33 (1987).

[26] G.W. Gibbons and C.M. Hull, Phys. Lett. 109B, 190 (1982).

[27] K. Becker, M. Becker, and A. Strominger, Phys. Rev. D 51, 6603 (1995).

[28] M. Anderson, F. Bonjour, R. Gregory, and J.M. Stewart, Phys. Rev. D 56, 8014 (1997). 